\title{
Термоэлектрические и термоэлектрокинетические явления в коллоидных растворах
}

\author{
(C) А.В. Сидоров ${ }^{1}$, В.М. Грабов ${ }^{2}$, А.А. Зайцев ${ }^{1}$, Д.В. Кузнецов ${ }^{1}$ \\ ${ }^{1}$ Елецкий государственный университет им. И.А. Бунина, \\ 399770 Елец, Россия \\ ${ }^{2}$ Российский государственный университет им. А.И. Герцена, \\ 191186 Санкт-Петербург, Россия \\ E-mail: dirnusir@mail.ru
}

Поступила в Редакцию 7 февраля 2019 г.

В окончательной редакции 10 февраля 2019 г.

Принята к публикации 14 фревраля 2019 г.

\begin{abstract}
На основе математического моделирования и эксперимента проводится анализ термоэлектрических и термоэлектрокинетических явлений в коллоидных растворах. Показано, что коллоидные частицы вносят значительный вклад в величину термоэлектрической и термоэлектрокинетической эдв и в большинстве случаев определяют знак коэффициента термоэлектрической эдс, прежде всего благодаря высоким значениям теплоты переноса коллоидных частиц по сравнению с ионами.
\end{abstract}

DOI: 10.21883/FTP.2019.06.47724.33

\section{1. Введение}

Как известно, термоэлектрические явления могут происходить не только в твердых телах, полупроводниках и металлах, но и в вязких электропроводящих средах, например, в растворах электролитов [1]. В последних вследствие их текучести также наблюдается открытый и экспериментально исследованный авторами термоэлектрокинетический эффект, относящийся к особому классу перекрестных явлений переноса, происходящих под действием трех термодинамических сил [2].

В этом случае в открытой для массопереноса системе, на которую наложен температурный градиент, формируется кинетическая неоднородность среды (рис. 1,b). В одной из ветвей системы скорость течения электропроводящей среды направлена вдоль температурного градиента, в другой - против него. Таким образом, обусловленные термодиффузией потоки заряженных частиц направлены в одном колене по течению, а в другом - против течения электропроводящей жидкости, что приводит в случае электрически разомкнутой цепи к формированию разности потенциалов, названной термоэлектрокинетической эдс.

В то же время особый класс вязких электропроводящих сред, в которых могут происходить термоэлектрические и термоэлектрокинетические явления, образуют коллоидные растворы. Известно, что коллоидные частицы при их добавлении в жидкость (дисперсионную среду), существенно меняют ее физико-химические свойства. Такие растворы характеризуются высокими значениями коэффициента Cope, прежде всего благодаря значительным величинам энтропий переноса $S_{i}$ коллоидных частиц, которые на 2-3 порядка превышают энтропию переноса ионов в растворах электролитов $[3,4]$. В работе [5] на примере коллоидного раствора гидрооксида железа показано, что величина термоэлектрической и термоэлектрокинетической эдс в коллоидных растворах может быть значительной. Поэтому в связи с широким распространением коллоидных растворов в природе, в частности в жидких системах живых организмов, исследование термоэлектрических и термоэлектрокинтических эффектов указанных растворов и установление микроскопических механизмов формирования соответствующих эдс является актуальной задачей.

Способы измерения термоэлектрической и термоэлектрокинетической эдс представлены на рис. 1, $a$ и $b$ соответственно и описаны в работе [5]. Корректное измерение разности потенциалов обеспечивалось применением хлорсеребряных электродов, обычно применяемых в электрохимических исследованиях [6].

В первом случае вязкая среда покоится в U-образной трубке 1, электрический нагреватель 2 создает вдоль
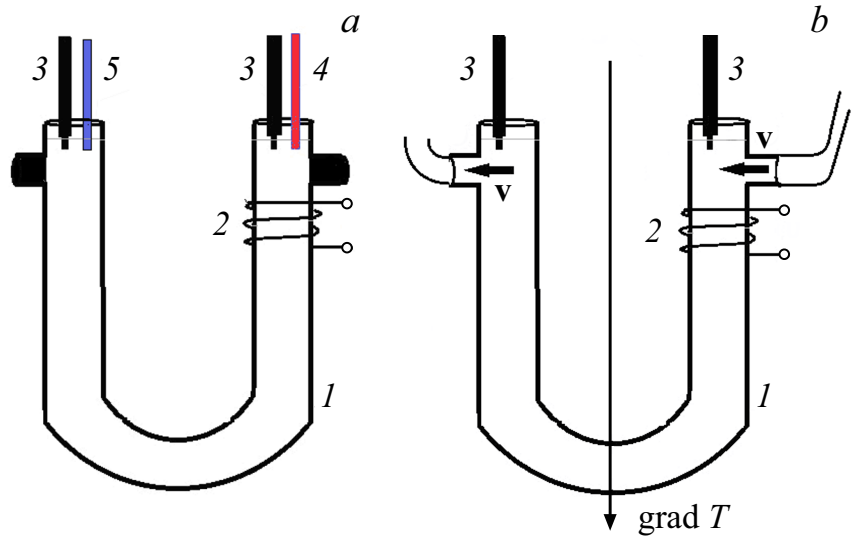

Рис. 1. Способ измерения термоэлектрической эдс $(a)$ и термоэлектрокинетической эдс $(b)$ в вязких электропроводящих средах. $1-\mathrm{U}$-образная трубка, заполненная электропроводящей жидкостью, 2 - электрический нагреватель, 3 - измерительные электроды, 4 - термодатчик, 5 - термометр. 
трубки перепад температур $\Delta T$. Тогда коэффициент термоэлектрической эдс определяется стандартным способом $\alpha=\Delta \varphi / \Delta T, \Delta \varphi-$ термоэлектрическая разность потенциалов.

В случае термоэлектрокинетической эдс (рис. 1,b) вертикальный градиент температуры создается путем подогрева U-образной трубки снизу, а электрический нагреватель 2 предназначен для выравнивания температур областей жидкости, контактирующих с измерительными электродами, с целью минимизации вклада термоэлектрической эдс в величину измеряемой термоэлектрокинетической эдс.

С помощью экспериментальных установок (рис. 1, $a$ и $b$ ) были исследованы термоэлектрический и термоэлектрокинетический эффекты в водных растворах электролитов. Величина термоэлектрической эдс указанных растворов меняется в широких пределах от десятков до сотен мкВ/K [7]. Наибольшими по абсолютной величине коэффициентами термоэлектрической эдс характеризуются растворы электролитов, кислоты и щелочи, распадающиеся на ионы, существенно различающиеся своими подвижностями. При исследовании термоэлектрокинетического эффекта в водных растворах электролитов были установлены основные его закономерности [7]: пропорциональность термоэлектрокинетической эдс величине перепада температуры, концентрации электролита, коэффициенту термоэлектрической эдс раствора, существенная зависимость от скорости течения среды. Знак термоэлектрокинетической эдс определяется знаком коэффициента термоэдс, направлением скорости течения среды и не зависит при этом от направления градиента температуры.

\section{2. Теория}

С целью теоретического описания термоэлектрического и термоэлектрокинетического эффектов в вязких электропроводящих средах и установления микроскопического механизма формирования термоэлектрокинетической эдс была построена математическая модель на основе уравнений физической кинетики [8].

В модели используются выражения для плотностей диффузионных потоков заряженных частиц электропроводящей среды Ј. Например, в случае раствора бинарного электролита [1]

$$
\begin{gathered}
\mathbf{J}_{+}=-\frac{c_{+} u_{+}}{F z_{+}}\left[z_{+} F \nabla(\varphi)+\frac{R T}{c_{+}} \nabla\left(c_{+}\right)+Q_{+} \frac{1}{T} \nabla(T)\right], \\
\mathbf{J}=\frac{c_{-} u_{-}}{F z_{-}}\left[z_{-} F \nabla(\varphi)+\frac{R T}{c_{-}} \nabla\left(c_{-}\right)+Q_{-} \frac{1}{T} \nabla(T)\right] .
\end{gathered}
$$

В уравнениях (1), (2) $F$ - постоянная Фарадея, $c_{ \pm}-$ объемные концентрации ионов, $u_{ \pm}-$их подвижности, $T$ - абсолютная температура, $Q_{ \pm}$- теплоты переноса ионов, $z_{ \pm}$- заряд частицы в единицах заряда электрона, $\varphi$ - электрический потенциал. В случае электрически разомкнутой цепи из соотношения $z_{+} \mathbf{J}_{+}+z_{-} \mathbf{J}_{-}=0$ может быть выражен градиент электрического потенциала, обусловленный наличием течения раствора и действия градиента температуры:

$$
\begin{aligned}
& \nabla(\varphi)=1 / F \\
& \times \frac{z_{+} a_{+}\left(-\frac{R T}{c_{+}}-\frac{Q_{+}}{T} \nabla(T)\right)+z_{-} a_{-}\left(-\frac{R T}{c_{-}}-\frac{Q_{-}}{T} \nabla(T)\right)}{z_{+}^{2} a_{+}+z_{-}^{2} a_{-}}
\end{aligned}
$$

В последнем соотношении для сокращения записи используются феноменологические коэффициенты $a_{ \pm}=c_{ \pm} u_{ \pm} / F z_{ \pm}$. Как следует из уравнения (3), разность электрических потенциалов определяется пространственными распределениями температуры и концентрации заряженных частиц, которые в случае нестационарного процесса, как, например, в условиях формирования термоэлектрокинетической эдс, могут быть определены из конвективных уравнений теплопроводности и диффузии [9]:

$$
\begin{aligned}
& \frac{\partial T}{\partial t}+v \frac{\partial T}{\partial x}=\chi \frac{\partial^{2} T}{\partial^{2} x}+\omega \\
& \frac{\partial c_{i}}{\partial t}=-\operatorname{div} \mathbf{J}_{i}-\operatorname{div}\left(c_{i} \mathbf{v}\right) .
\end{aligned}
$$

В уравнениях (4),(5) v - скорость течения среды, $\chi-$ коэффициент температуропроводности, $\omega-$ мощность источников и стоков теплоты; для бинарного раствора электролита индексы $i$ в уравнениях (5) относятся к положительным и отрицательным ионам. Таким образом, система уравнений (1)-(5) может быть использована для описания как термоэлектрического $(v=0)$, так и термоэлектрокинетического $(v \neq 0)$ эффектов. Решение полученной системы осуществляется методом конечных разностей, как описано в [8].

Таким образом, в основе модели лежат уравнения физической кинетики в гидродинамическом приближении, при этом для плотностей диффузионных потоков заряженных частиц используются универсальные соотношения неравновесной термодинамики Онзагера, которые остаются справедливыми для сред любой физической природы. Так, например, для твердого электронного проводника при наложении температурного градиента и в случае отсутствия градиентов концентрации плотность диффузионного потока электронов [1] есть

$$
\mathbf{J}=-\beta F \operatorname{grad}(\varphi)+\frac{\gamma}{T} \operatorname{grad}(T)
$$

причем феноменологические коэффициенты $\beta=\sigma / F^{2}$, $\gamma=\beta Q_{-}=\sigma Q_{-}^{e} / F^{2}$ выражаются через удельную электропроводность $\sigma$ и теплоту переноса электронов $Q_{-}^{e}$. В случае электрически разомкнутой цепи (плотность тока равна нулю) соотношение (6) позволяет получить выражение для термоэлектрической разности потенциалов в твердом электронном проводнике 


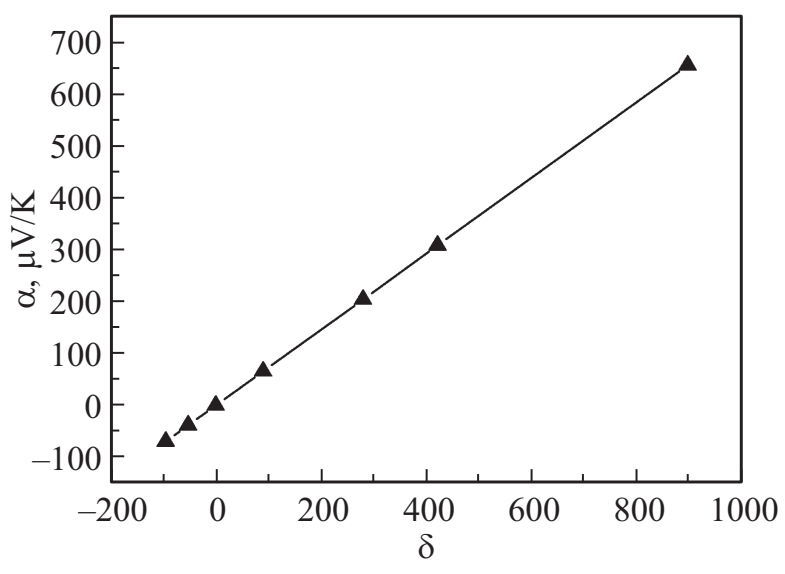

Рис. 2. Расчетная зависимость коэффициента термоэдс $\alpha$ коллоидного раствора от безразмерного фактора $\delta=\left(Q_{k} u_{k}-Q_{i} u_{i}\right) / Q_{i} u_{k}$.

$\nabla(\varphi)=\left(Q_{-} / F T\right) \nabla(T)$, которое показывает, что коэффициент термоэлектрической эдс для такой электропроводящей среды определяется только теплотой переноса электронов. Если определить, согласно [10], энтропию переноса частиц соотношением

$$
S_{i}=\frac{Q_{i}}{T}
$$

окончательно получим, что $\nabla(\varphi)=\left(S_{-}^{e} / F\right) \nabla(T)$. Отметим что соотношение (3) для вязкой электропроводящей среды также может быть переписано через энтропии переноса ионов. Универсальность используемого подхода позволяет обобщить его на случай коллоидных растворов. В коллоидных растворах в простейшем случае имеем два типа заряженных частиц: крупные коллоидные частицы, имеющие заряд за счет адсорбции на своей поверхности одноименно заряженных ионов электролита, и противоионы, которые образуют диффузный слой вокруг ядра коллоидной частицы, частично экранируя ее поле. Анализ соотношения (3) показывает, что в случае бинарной электропроводящей среды термоэлектрическая разность потенциалов определяется конкурирующими вкладами противоположно заряженных частиц, имеющих различные величины подвижностей $u_{ \pm}$ и энтропии переноса $S_{i}=Q_{i} / T$. Энтропии переноса коллоидных частиц значительно выше энтропии переноса ионов, а их подвижности приблизительно на два порядка ниже [11]. Величина термоэлектрическsой эдс коллоидного раствора при этом во многом определяется безразмерным параметром

$$
\frac{Q_{k}}{Q_{i}}-\frac{u_{i}}{u_{k}}=\frac{Q_{k} u_{k}-Q_{i} u_{i}}{Q_{i} u_{k}},
$$

где $Q_{k}, u_{k}, Q_{i}, u_{i}$ - теплоты переноса и подвижности коллоидных частиц и ионов соответственно. Знак термоэлектрической эдс будет определяться коллоидными частицами, если выполняется неравенство $Q_{k} u_{k}>Q_{i} u$.
Данные предположения подтверждаются расчетом на основе уравнений модели (1)-(5). В расчете предполагается, что коллоидные частицы имеют положительный заряд, ионы - отрицательный, подвижности и теплоты переноса ионов соответствуют ионам хлора [1]. Величина подвижности коллоидной частицы на 2 порядка ниже подвижности иона, величина теплоты переноса коллоидной частицы является переменной величиной, при этом отношение $\delta=\left(Q_{k} u_{k}-Q_{i} u_{i}\right) / Q_{i} u_{k}$ меняется в пределах от -100 до 900. Верхняя граница соответствует ситуации, когда теплота переноса коллоидной частицы на 3 порядка выше теплоты переноса ионов, что соответствует максимально возможному отношению теплоты переноса коллоидных частиц и ионов, полученному в экспериментах с коллоидными растворами [3,4]. На рис. 2 изображена расчетная зависимость величины термоэлектрической эдс от величины $\delta=\left(Q_{k} u_{k}-Q_{i} u_{i}\right) / Q_{i} u_{k}$. Коэффициент термоэлектрической эдс оказывается прямо пропорциональным фактору $\delta$ и меняется в широких пределах; если $Q_{k} u_{k} \approx Q_{i} u_{i}$, коэффициент термоэлектрической силы коллоидного раствора будет близок к нулю.

\section{3. Экспериментальные результаты и их обсуждение}

На основе описанной выше экспериментальной установки (рис. 1, $a, b)$ были проведены измерения термоэлектрической и термоэлектрокинетической эдс коллоидных растворов. Как показывает теоретический анализ, величина термоэлектрической эдс коллоидных растворов должна меняться в пределах от единиц до нескольких сотен мкВ, ее величина и знак определяются прежде всего соотношениями между теплотами переноса и подвижностями коллоидных частиц и ионов раствора. Поэтому представляет интерес измерение термоэлектрической эдс в коллоидных растворах различной природы.

На рис. 3,4 представлены экспериментальные зависимости термоэлектрической разности потенциалов от перепада температур для гидрофобного коллоидного раствора гидроксида железа (III), полученного в реакции гидролиза 2\%-го раствора хлорного железа $\mathrm{FeCl}_{3}$ [12] (рис. 3) и гидрофобного золя берлинской лазури, полученного методом адсорбционной пептизации гексацианоферрата калия $\mathrm{K}_{4} \mathrm{Fe}(\mathrm{CN})_{6}$ в избытке $\mathrm{FeCl}_{3}$ (рис. 4) [12].

Определенные из серии экспериментов величины коэффициента термоэлектрической эдс указанных растворов составляют $\alpha=+(350 \pm 30)$ мкВ/K для золя гидроксида железа и $\alpha=+(22 \pm 10)$ мкВ/К для берлинской лазури, т.е. результаты различаются на порядок. Знак коэффициента $\alpha$ совпадает со знаком заряда коллоидных частиц. Коллоидный раствор берлинской лазури является неустойчивым, коллоидные частицы в нем коагулируют и спустя несколько суток выпадают в осадок. Коэффициент термоэдс полученного раствора, из которого удалены коллоидные частицы, при этом становится практически равным нулю, т.е. уменьшается на 
Результаты измерения коэффициента термоэлектрической эдс в коллоидных растворах

\begin{tabular}{|c|c|c|c|c|}
\hline Коллоидный раствор & $\begin{array}{c}\text { Коэффициент } \\
\text { термоэлектрической эдс, } \\
\text { мкВ/K }\end{array}$ & $\begin{array}{c}\text { Знак заряда } \\
\text { коллоидной } \\
\text { частицы }\end{array}$ & $\begin{array}{c}\text { Потенциало- } \\
\text { определяющие } \\
\text { ионы }\end{array}$ & Противоионы \\
\hline $\begin{array}{l}\text { Гидроксид железа (III) } \\
\text { Берлинская лазурь } \\
\text { Иодид серебра } \\
\text { Иодид серебра } \\
\text { Альбумин человеческий }\end{array}$ & $\begin{array}{c}+350 \\
+22 \\
\text { В пределах погрешности } \\
-46 \\
-74\end{array}$ & $\begin{array}{l}+ \\
+ \\
+ \\
- \\
-\end{array}$ & $\begin{array}{l}\mathrm{FeO}^{+} \\
\mathrm{Fe}^{3+} \\
\mathrm{Ag}^{+} \\
\mathrm{I}^{-}\end{array}$ & $\begin{array}{c}\mathrm{Cl}^{-} \\
\mathrm{Cl}^{-} \\
\mathrm{NO}_{3}^{-} \\
\mathrm{K}^{+}\end{array}$ \\
\hline
\end{tabular}

порядок, что свидетельствует о значительности вклада коллоидных частиц в величину термоэдс в этом случае.

Как показывают результаты экспериментов в водных растворах электролитов, величина термоэлектрокинети-

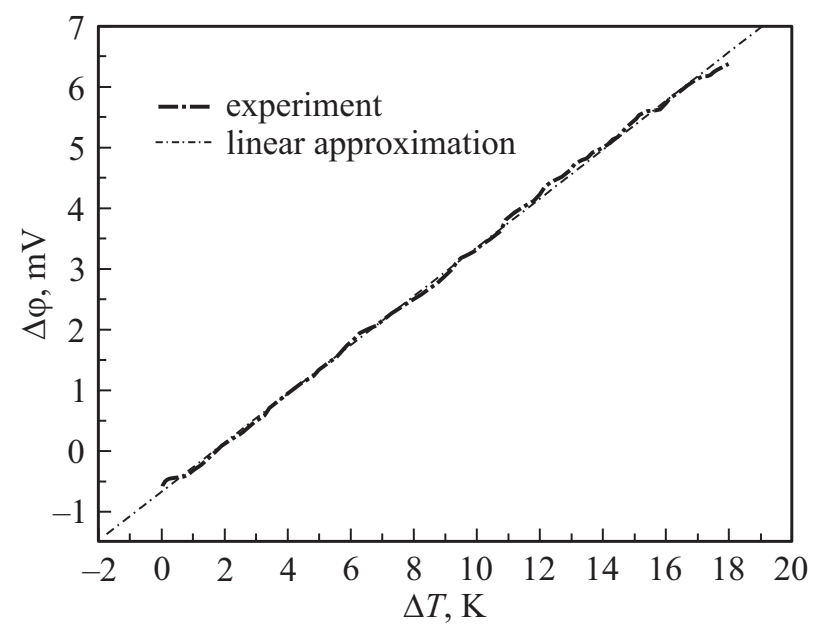

Рис. 3. Зависимость термоэлектрической разности потенциалов $\Delta \varphi$ коллоидного раствора $\mathrm{Fe}(\mathrm{OH})_{3}$ от разности температур $\Delta T$. Мицелла заряжена положительно. Средняя температуpa $302 \mathrm{~K}$.

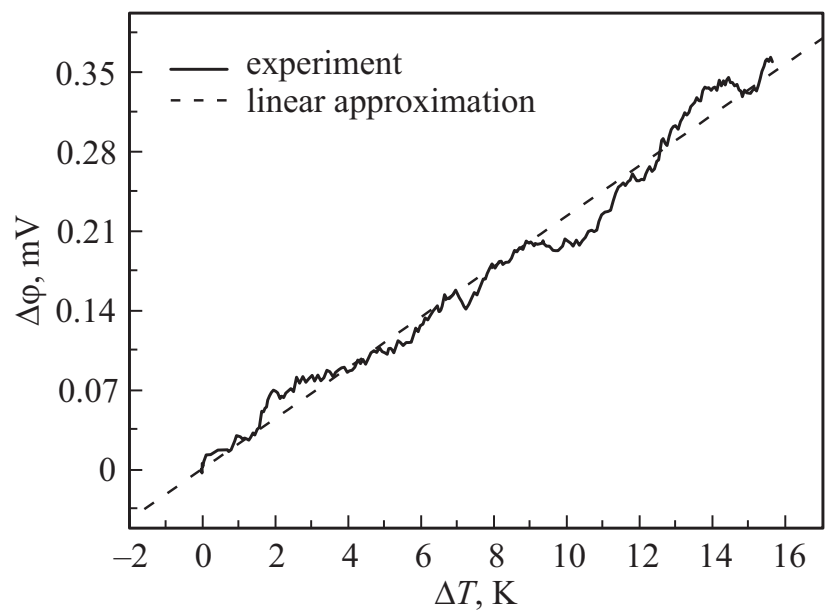

Рис. 4. Зависимость термоэлектрической разности потенциалов коллоидного раствора берлинской лазури от разности температур. Мицелла заряжена положительно. Средняя температура $302 \mathrm{~K}$. ческой эдс оказывается прямо пропорциональной коэффициенту термоэлектрической эдс. Величина коэффициента термоэлектрической эдс коллоидного раствора гидроксида железа сравнима с величиной коэффициента термоэдс кислот и щелочей, измеренная в работе [5] величина термоэлектрокинетической эдс данного раствора также оказывается заметной, $(0.5 \pm 0.3)$ мВ. Таким образом, в коллоидных растворах термоэлектрокинетический эффект может играть значительную роль.

В таблице приведены сводные данные по измерению коэффициента термоэлектрической эдс в различных коллоидных растворах.

Большинство растворов, представленных в таблице, относится к неорганическим гидрофобным золям, за исключением альбумина, который является раствором смеси высокомолекулярных белков в дистиллированной воде и основным белковым компонентом человеческой крови. Благодаря наличию на поверхности его молекулы большого числа отрицательно заряженных групп в дистиллированной воде молекулы альбумина несут отрицательный заряд [13].

Полученные результаты (см. таблицу) показывают, что, действительно, величина коэффициента термоэлектрической силы коллоидных растворов меняется в диапазоне от нескольких единиц до нескольких сотен мкВ/K, знак заряда коэффициента $\alpha$ в основном определяется знаком заряда коллоидных частиц.

Как известно, коллоидные частицы сами по себе не имеют заряда, они его приобретают в растворе, адсорбируя на своей поверхности присутствующие там ионы. В зависимости от преобладания компонентов можно получать коллоидные частицы, заряженные положительно или отрицательно, тем самым управляя термоэлектрическими свойствами коллоидных растворов. Так, например, в реакции двойного обмена при получении коллоидного раствора иодида серебра согласно реакции $\mathrm{AgNO}_{3}+\mathrm{KI} \rightarrow \mathrm{KNO}_{3}+\mathrm{AgI} \downarrow$ в случае преобладания иодида калия получается мицелла иодида серебра, заряженная отрицательно, коэффициент термоэлектрической силы такого раствора равен -46 мкВ/К (см. таблицу). В случае преобладания в исходных компонентах нитрата серебра коллоидные частицы адсорбируют положительные ионы $\mathrm{Ag}^{+}$, противоионами являются нитратионы $\mathrm{NO}_{3}^{-}$. Коэффициент термоэдс при этом практически становится равным нулю (см. таблицу). 


\section{4. Заключение}

Таким образом, полученные экспериментальные результаты и результаты моделирования термоэлектрического и термоэлектрокинетического эффектов свидетельствуют о том, что величина термоэлектрической и термоэлектрокинетической эдс помимо значений термодинамических сил зависит от соотношения между микроскопическими характеристиками заряженных частиц, их подвижностями и теплотами переноса.

В коллоидных растворах наблюдается уникальная ситуация, когда один из типов зарядов (коллоидные частицы) практически не вносят вклада в электрическую проводимость и в то же время вносят значительный вклад, превышающий в ряде случаев вклад ионов, в величину термоэлектрической силы раствора. Данный факт обусловлен экстремально высокими значениями теплоты переноса коллоидных частиц по сравнению с ионами. Величина и знак коэффициента термоэлектрической силы в коллоидных растворах определяются конкурирующими вкладами ионов и коллоидных частиц, которые можно оценить с помощью безразмерного фактора $\delta=\left(Q_{k} u_{k}-Q_{i} u_{i}\right) /\left(Q_{i} u_{k}\right)$.

Из результатов экспериментов также следует, что изменяя состав дисперсионной среды, можно сравнительно легко управлять термоэлектрическими свойствами коллоидных растворов.

Таким образом, изучение термоэлектрических и термоэлектрокинетических явлений в коллоидных раствоpax является важной задачей прежде всего благодаря широкому распространению таких систем в природе, в частности в жидких системах живых организмов. Учет этих явлений необходим для понимания роли термоэлектромагнитных процессов в живых организмах.

Работа выполнена при поддержке РФФИ и администрации Липецкой области, грант № 17-42-480248.

\section{Список литературы}

[1] Р. Хаазе. Термодинамика необратимых процессов (М., Мир, 1967).

[2] В.М. Грабов, А.А. Зайцев, Д.В. Кузнецов, А.В. Сидоров, И.В. Новиков Вестн. Московского гос. техн. ун-та им. Н.Э. Баумана. Серия: Естественные науки, 3, 112 (2008).

[3] J. Lenglet et al. Phys. Rev. E, 65, 031408 (2002).

[4] R. Piazza, A. Parola. J. Phys.: Condens. Matter, 20, 153102 (2008).

[5] В.М. Грабов, А.А. Зайцев, Д.В. Кузнецов, А.В. Сидоров. ЖТФ, 88 (10), 1462 (2018).

[6] Б.Д. Степин. Техника лабораторного эксперимента в химии (М., Химия, 1999).

[7] В.М. Грабов, А.А. Зайцев, Д.В. Кузнецов. Термоэлектричество, 1, 43 2010).

[8] А.В. Сидоров, В.М. Грабов, А.А. Зайцев, Д.В. Кузнецов, Д.А. Нарциссов. Вестн. Воронежского гос. техн. ун-та, 13 (5), 113 (2017).
[9] Д.А. Франк-Каменецкий. Основы макрокинетики. Дифбузия и теплопередача в химической кинетике (Долгопрудный, Издат. дом „Интеллект“, 2008).

[10] С.Р. де Грот. Термодинамика необратимых процессов (М., Гос. изд. техн.-теорет. лит., 1956).

[11] С.С. Духин. Электропроводность и электрокинетические свойства коллоидных систем (Киев, Наук. думка, 1975).

[12] С.С. Воюцкий. Курс коллоидной химии (М., Химия, 1974).

[13] У. Льюис, Л. Скуайрс, Д. Брутон. Химия коллоидных и аморбных веществ (М., Гос. изд-во иностр. лит., 1948).

Редактор Л.В. Шаронова

\section{Thermoelectric and thermoelectrokinetic phenomena in colloidal solutions}

\author{
A.V. Sidorov' ${ }^{1}$, V.M. Grabov ${ }^{2}$, A.A. Zaitsev' ${ }^{1}$, \\ D.V. Kuznetsov ${ }^{1}$ \\ ${ }^{1}$ Bunin State University, \\ 399770 Yelets, Russia \\ ${ }^{2}$ Herzen State Pedagogical University of Russia, \\ 191186 St. Petersburg, Russia
}

Abstract On the basis of mathematical modeling and experiment the analysis of thermoelectric and thermoelectrokinetic phenomena in colloidal solutions is carried out. It is shown that colloidal particles make a significant contribution to the value of thermoelectric and thermoelectrokinetic emf and in most cases determine the sign of the thermoelectric emf coefficient, primarily due to the high values of the heat transfer of colloidal particles compared to ions. 